\title{
Spontaneous coronary artery dissection in the postpartum period
}

\author{
B.J.L. Van den Branden, W.A.J. Bruggeling, H.M.A Corbeij, P.H.J.M. Dunselman
}

Spontaneous coronary artery dissection is a very uncommon cause of acute coronary syndrome. It occurs predominantly in young to middle-aged women during or after pregnancy. The aetiology remains uncertain. Possible factors are hormonal changes, haemodynamic stress and changes in autoimmune status. In case of single-vessel dissection and normal blood flow, conservative treatment often leads to complete angiographic resolution. This case report describes the clinical presentation, diagnosis and therapy of spontaneous coronary artery dissection in a 37-year-old woman in the postpartum period. (Neth Heart J 2008;16: 412-4.)

Keywords: postpartum, coronary artery, dissection

A 37-year-old woman with a history of hypothyroidism and mild chronic obstructive pulmonary disease (COPD) was referred to our department because of a squeezing chest discomfort, starting at rest five hours before presentation, radiating to both arms and the interscapular region. There was no response to nitroglycerine. She had never experienced this pain before. She had no known risk factors for atherosclerotic disease and no history of any connective tissue disorder. She did not smoke and did not use alcohol or drugs. Three weeks before presentation she uneventfully delivered a healthy baby (G4P4). The course of her pregnancy had been uncomplicated.

\section{B.J.L. Van den Branden}

Department of Cardiology, Amphia Hospital, Breda; currently working at St Antonius Hospital, Nieuwegein, the Netherlands W.A.J. Bruggeling

H.M.A Corbeij

P.H.J.M. Dunselman

Department of Cardiology, Amphia Hospital, Breda, the Netherlands

Correspondence to: W.A.J. Bruggeling

Department of Cardiology, Amphia Hospital, PO Box 90158, 4800 RK, Breda, the Netherlands

E-mail: wbruggeling@amphia.nl
On physical examination her blood pressure was $140 / 85 \mathrm{mmHg}$ and her heart rate 92 beats/min. Auscultation of heart and lung fields was normal. Physical examination revealed no signs of heart failure or Marfan's syndrome.

Chest X-ray was normal. Her ECG showed sinus rhythm, 63 beats/min, left axis deviation and loss of $\mathrm{R}$ amplitude in the precordial leads $\mathrm{V}_{1}$ to $\mathrm{V}_{4}$ with minimal elevation of the $S T$ segment in leads $V_{5}$ and $\mathrm{V}_{6}$ (figure $\mathrm{l}$ ).

Laboratory measurements on admission showed a normal C-reactive protein of $3 \mathrm{mg} / \mathrm{l}$, normal creatinine $60 \mu \mathrm{mol} / \mathrm{l}$ and elevated cardiac markers: creatinine kinase $355 \mathrm{U} / 1$, rising to a maximum of $748 \mathrm{U} / \mathrm{l}$, troponin $\mathrm{T}$ of $0.41 \mu \mathrm{g} / \mathrm{l}$, rising to a maximum of 2.1 $\mu \mathrm{g} / \mathrm{l}$, aspartate aminotranferase $68 \mathrm{U} / \mathrm{l}$ and lactate dehydrogenase $323 \mathrm{U} /$. D-dimer was normal.

Total cholesterol was $7.2 \mathrm{mmol} / \mathrm{l}$, high-density lipoprotein cholesterol $1.3 \mathrm{mmol} / \mathrm{l}$ and low-density lipoprotein cholesterol $5.46 \mathrm{mmol} / \mathrm{l}$.

She was admitted under the diagnosis of subacute inferolateral myocardial infarction and was treated with $\beta$-blockers, low-molecular-weight heparin, clopidogrel and acetylsalicylic acid. Transthoracic echocardiography showed a normal right and left ventricular function with mild left ventricular hypertrophy.

One week after referral she underwent cardiac catheterisation (this was delayed because of fever of unknown origin). Coronary angiogram showed dissection of the circumflex coronary artery, which extended through the first left posterolateral branch (figure 2). The other coronary arteries showed no abnormalities.

We proposed conservative treatment. She remained free of cardiac symptoms. A control angiogram was performed four months after discharge. This showed some improvement and incomplete resolvement of the dissection (figure 3).

Myocardial SPECT scintigraphy revealed an irreversible perfusion defect at the apical inferior wall of the left ventricle, suggestive of an old infarction. There was no residual ischaemia, the left ventricular function was normal. 


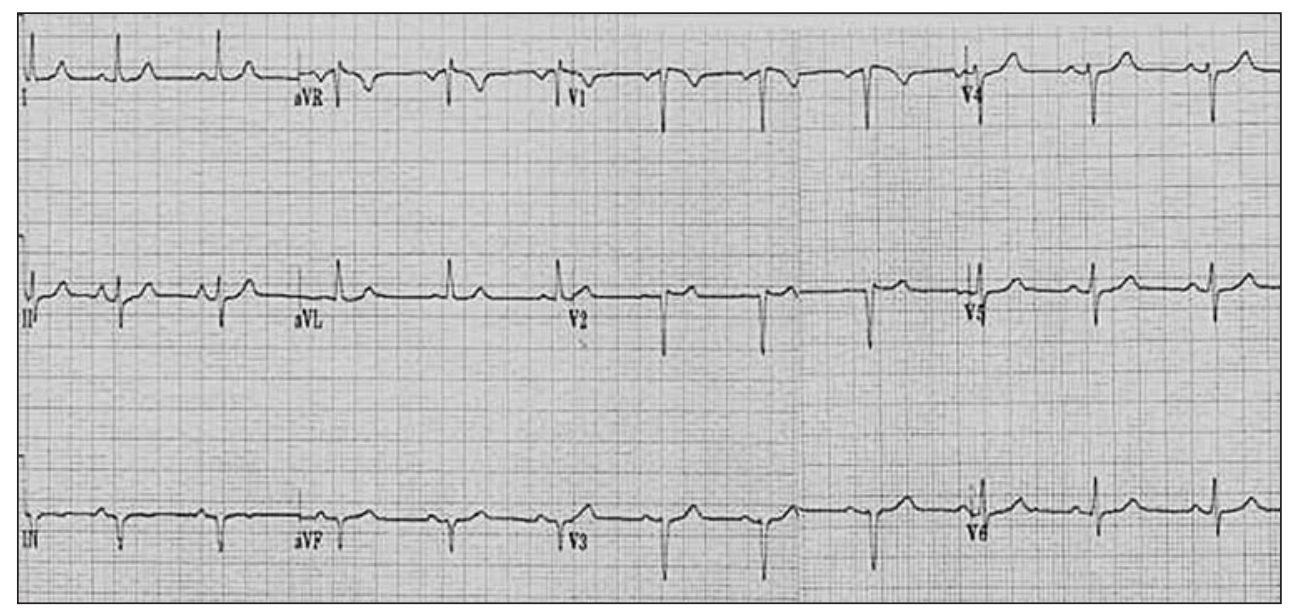

Figure 1. Electrocardiogram.

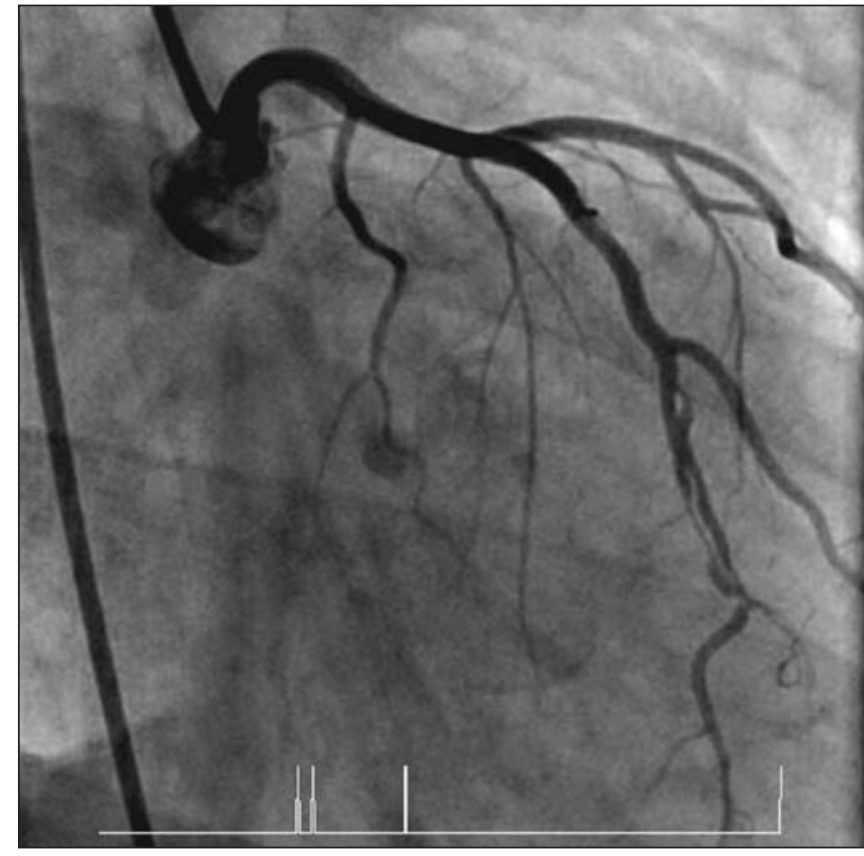

Figure 2. Left coronary artery with a dissection in the right circumflex and posterolateral branch.

\section{Discussion}

Acute myocardial infarction (AMI) is uncommon under the age of 40 years, especially among women. This group only accounts for about $0.7 \%$ of all cases of AMI. ${ }^{1}$ Primary non-traumatic spontaneous coronary artery dissection is an uncommon cause of AMI in the younger age group. It was first described in the case of a 42 -year-old woman in $1931 .{ }^{2}$ More than 150 cases have been reported in the literature so far. It is usually seen in young, apparently healthy women, without overt cardiovascular risk factors. Approximately one in four female patients with spontaneous coronary artery dissection are in the peripartum period, most commonly in the third trimester of pregnancy or in the early postpartum period.

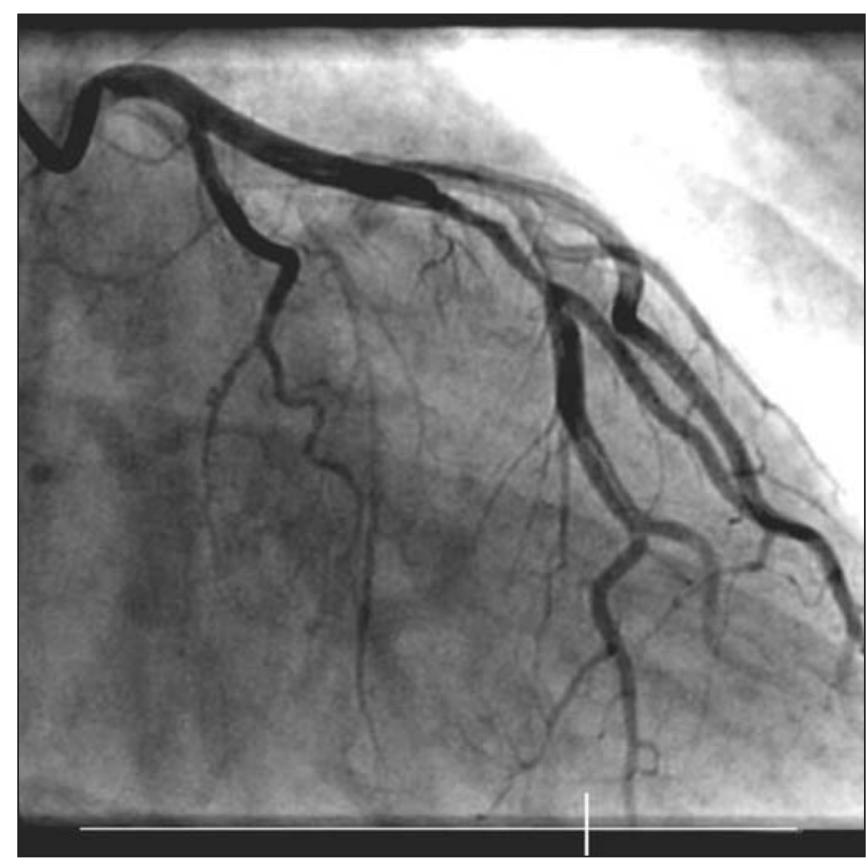

Figure 3. Partial resolution of the dissected part in the right circumflex artery.

On the basis of epidemiological data, this condition occurs in one in 20,000 to 30,000 deliveries.

In general, this disease is associated with high mortality, about $50 \%$ at presentation. ${ }^{3}$ There is an $85 \%$ survival rate for patients who survive the acute phase. ${ }^{4}$ Sudden death without preceding myocardial ischaemia is a frequent mode of presentation. The overall mortality in those patients who do present with myocardial infarction exceeds $70 \%$. This is why it is usually recognised at post-mortem examination.

Spontaneous coronary artery dissection occurs in normal coronary arteries.

In women, there is a predilection for the left coronary artery system, whereas in men the right coronary artery is affected in about two thirds of the cases. ${ }^{5}$ 
The aetiology and pathogenesis of spontaneous coronary artery dissection remains poorly understood. The high incidence in the peripartum period indicates the possibility that hormonal changes weaken the arterial wall and predispose it to rupture or dissection. Another possible pathophysiological factor is the haemodynamic stress during pregnancy. Heefner hypothesised that this will lead to an initial intimal rupture. ${ }^{6}$ This is followed by delayed bleeding in the tunica media caused by the clotting changes that occur during pregnancy.

In the absence of an intimal tear, primary disruption of the vasa vasorum and subsequent haemorrhage into the media of the arterial wall has been proposed as an underlying mechanism of spontaneous coronary artery dissection. A case report described the correlation between antiphospholipid antibodies and spontaneous coronary artery dissection in pregnancy. ${ }^{7}$

Additionally, bromocriptine, which is used for lactation inhibition, has been related to postpartum myocardial infarction. It may initiate coronary spasm leading to the artery wall dissection. ${ }^{8}$

Several authors noted the presence of periadventitial inflammatory infiltrates. Such infiltrates could induce fragility of the arterial wall secondary to the release of lytic proteases. ${ }^{9}$ Other reported possible causes of spontaneous coronary artery dissection, seen without pregnancy, are cocaine abuse, intense physical exercise and the use of oral contraceptives. ${ }^{10,11}$ There is also a possible correlation with Marfan's syndrome. Cystic medial necrosis and other defects in collagen metabolism have also been described in some patients. ${ }^{5}$

Therapy should depend on the persistence of myocardial ischaemia, the area at risk and the number of vessels involved. In patients who survive the acute phase and who subsequently remain asymptomatic, medical management is safe and compatible with good longterm survival. Thrombolytic therapy could lyse the compressing clot, but it could also contribute to the expansion of an intramural haematoma. Thrombolysis may be useful in arteries where the intramural haematoma is compressing the true lumen, allowing the latter to re-expand. ${ }^{12}$ Stenting is the considered therapy in case of a well-localised dissected lesion in a single vessel not involving the left main stem. ${ }^{13}$ On the other hand it will be difficult to stent a complicated dissection involving thrombus and a nearly occluded lumen. ${ }^{14}$ In case of multivessel or left main stem involvement, surgical revascularisation seems the most controlled strategy, although the anastomosis of the graft on a dissected coronary artery is hazardous. ${ }^{15} \mathrm{In}$ patients with ongoing myocardial ischaemia or in patients refractory to medical treatment, bypass surgery also is the most appropriate therapy.

\section{Conclusion}

Spontaneous coronary artery dissection is a rare but important cause of myocardial ischaemia and infarction in young, healthy women, without classical coronary risk factors. One in four cases are seen in the peripartum period. Sudden death, without ischaemia, is a frequent mode of presentation. The overall mortality in those patients who present with myocardial infarction is in excess of $70 \%$.

Immediate coronary angiography is essential to establish an early diagnosis and allowing a therapeutic decision.

Our case report confirms that, when a single coronary vessel is affected and the lesion is not attractive to stent, medical treatment is safe.

\section{References}

1 Badui E, Enciso R. Acute myocardial infarction during pregnancy and puerperium : a review. Angiology 1996;47:739-56

2 Pretty HC. Dissecting aneurysm of coronary artery in a woman aged 42. BMJ 1931;1:667

3 Jorgensen MB, Aharonian V, Mansukhani P, et al. Spontaneous coronary dissection : a cluster of cases with this rare finding. Am Heart J 1994; 127:1382-7

4 Engelman DT, Thayer J, Derossi J, et al. Pregnancy related coronary artery dissection: a case report and collective review. Conn Med 1993;57:135-9

5 Conraads VM, Vorlat A, Colpaert CG, et al. Spontaneous dissection of three major coronary arteries subsequent to cystic medial necrosis. Chest 1999;116:1473-5.

6 Heefner WA. Dissection hematoma of the coronary artery: a possible complication of oral contraceptive therapy. JAMA 1973;223 550-1.

7 Krishnamurthy M, Desai R, Patel H. Spontaneous coronary artery dissection in the postpartum period: association with antiphospholipid antibody. Heart 2004;90:e53

8 Hopp L, Weise AB, Iffy L. Acute myocardial infarction in a healthy mother using bromocriptine for milk suppression. Can J Cardiol 1996;12:415-8

9 Siegel RJ, Koponen M. Spontaneous coronary artery dissection causing sudden death: mechanical arterial failure or primary vasculitis? Arch Pathol Lab Med 1994;118:196-8

10 Jaffe BD, Broderick TM, Leier CV. Cocaine-induced coronary artery dissection. N Engl J Med 1994;330:510-1

11 Sherrid MV, Mieres J, Mogtader A, et al. Onset during exercise of spontaneous coronary artery dissection and sudden death: occurrence in a trained athlete: case report and review of prior cases. Chest 1995; 108:284-7

12 Missouris CG, Ring A, Ward D. A young woman with chest pain. Heart 2000;84:e12.

13 Porras MC, Ares MA, Zucco J. Intracoronary stenting for postpartum coronary artery dissection. Ann Intern Med 1998;128:873

14 Hong MK, Satler LF, Mintz GS. Treatment of spontaneous coronary artery dissection with intracoronary stenting. Am Heart J 1996;132:2000-2

15 Thislewaite PA. Surgical management of spontaneous left main coronary artery dissection. Ann Thorac Surg 1998;45:258-60. 